\title{
Telemedicina tra clinica e nutrizione nella Malattia Renale Cronica (MRC): appunti di esperienze al femminile
}

\author{
Anna Laura Fantuzzi ${ }^{1}$, Elisa Berri ${ }^{2}$, Lida Tartaglione ${ }^{3,4}$, Monica Prampolini ${ }^{5}$, Rossella Giannini ${ }^{6}$, Sara Dominjanni ${ }^{7}$, Silvia Porreca $^{8}$ \\ ${ }^{1}$ Dietista già Coordinatore Nutrizione e Dietetica Aziendale, AUSL Modena, Comitato Scientifico ASAND (Associazione Tecnico Scientifica \\ dell'Alimentazione, Nutrizione e Dietetica) - Italy \\ ${ }^{2}$ Dietista, Nutrizione e Dietetica Aziendale, Azienda Unità Sanitaria Locale Modena - Italy \\ ${ }^{3}$ Dirigente Medico, UOC Nefrologia e Dialisi, ASL RM5 - P.O. Tivoli (RM) - Italy \\ ${ }^{4}$ Dirigente Medico, Dipartimento Medicina di Traslazione e di Precisione, Sapienza Università di Roma, Roma - Italy \\ ${ }^{5}$ Dietista, libero professionista, Modena - Italy \\ ${ }^{6}$ Dietista, SSD Malattie del Metabolismo e Nutrizione Clinica, Azienda Ospedaliero-Universitaria Policlinico di Modena, Modena - Italy \\ ${ }^{7}$ Dirigente Medico, UOC Nefrologia e Dialisi Ospedale Sant'Eugenio, AUSL Roma 2, Roma - Italy \\ ${ }^{8}$ Dirigente Medico, U.O. di Nefrologia e Dialisi, P.O. Della Murgia “F. Perinei”, Altamura (BA), ASL BA - Italy
}

\begin{abstract}
Telemedicine between clinical evaluation and nutrition in Chronic Kidney Disease (CKD): experience from the feminine point of view

In Italy, starting from 2015, the date of publication of the guidelines on the 2014-2020 Digital Growth Strategy, technological evolution in medicine has provided encouraging results. The term telemedicine is now in common use, and it is also indicated at an encyclopedic level as "the set of technical and health care monitoring tools, created through systems designed to provide quick access to both medical specialists and patients, regardless of the place where they are respectively located". Nowadays, in the COVID era, the need for social distancing has highlighted the contribution of digital technology to healthcare in terms of access to care and healthcare spending. With regard to chronic kidney disease, telemedicine has always proved essential in improving patients' quality of life, as in the case of peritoneal dialysis. The remote management and monitoring of patients undergoing peritoneal dialysis has not only proved to radically change and improve patients' quality of life in replacement therapy, but has also reduced costs. There are some situations in which telemonitoring can guarantee the maintenance of an adequate quality of life for patients in the different stages of the disease. Thanks to the constant commitment of some groups of professionals where the largest part is represented by the "pink quota", it was possible to experiment and implement evaluation protocols for the renal patient in conservative and replacement treatment in some of the most central aspects of care: medical and dietary therapy.
\end{abstract}

Keywords: Kidney Diseases, Nutrition, Telemedicine, Women in Medicine

\section{Introduzione}

L'emergenza sanitaria da COVID-19 che tutto il mondo si è trovato ad affrontare ha messo in risalto in modo chiaro l'importanza del "monitoraggio della salute" a distanza.

La necessità di trasformare la sanità in un sistema più efficiente e focalizzato sul paziente nell'era dell'innovazione digitale è evidente e, in questo periodo a elevata complessità, tutti hanno sentito l'esigenza di percorsi di cura condotti

Received: January 23, 2021

Accepted: February 3, 2021

Published online: February 20, 2021

Indirizzo per la corrispondenza:

Anna Laura Fantuzzi

Via Bismantova 4

42019 Scandiano (RE) - Italy

annalaurafantuzzi@gmail.com in un rapporto professionista sanitario-paziente sempre più integrato, ma a distanza, attraverso strumenti telematici.

In Italia, a partire dal 2015, data di pubblicazione delle Linee Guida sulla Strategia per la Crescita Digitale 2014-2020 (1), l'evoluzione tecnologica in medicina ha fornito risultati incoraggianti. Il termine telemedicina è ormai di uso comune, tanto da essere indicato anche a livello enciclopedico come "I'insieme delle tecniche e degli strumenti di monitoraggio e di assistenza sanitaria, realizzato mediante sistemi atti a fornire un rapido accesso sia ai medici specialisti che ai pazienti, prescindendo dal luogo in cui essi sono rispettivamente situati" (2).

La telemedicina, a differenza di quello che si può pensare, non ha una storia recente, comparendo negli USA già nel 1950. In quegli anni, la finalità era quella di monitorare il sistema cardiocircolatorio degli astronauti nello spazio, per assicurare un'assistenza sanitaria quanto più efficace possibile a distanza. In Italia, le prime esperienze di trasmissione di 
segnali biomedicali vennero realizzate nel 1970 dalla Facoltà di Medicina dell'Università degli Studi di Roma "La Sapienza" con la sperimentazione di un prototipo di Cardiotelefono (3). In molti paesi europei, la telemedicina è molto diffusa ormai da diversi anni e, in alcuni casi, è sostenuta da interventi normativi, da documenti strategici e da progetti a livello nazionale. Per esempio, in Svezia, già nel 2008 la telemedicina era in uso in oltre il $75 \%$ degli Ospedali e le principali aree applicative erano la Televisita (medico-paziente), il telemonitoraggio e il teleconsulto radiologico (4).

In termini strettamente pratici, la telemedicina dovrebbe consentire, oltre alla possibilità di una cartella clinica elettronica costantemente aggiornata e accessibile al personale sanitario, l'ospedalizzazione domiciliare e la gestione a distanza dei pazienti attraverso il monitoraggio di alcuni parametri clinici (p. es., peso, frequenza cardiaca, frequenza respiratoria, ecc.). Sappiamo come, nelle malattie croniche, come la malattia renale, il controllo di alcuni parametri possa incidere significativamente sulla riduzione degli accessi ospedalieri e, di conseguenza, della spesa sanitaria (5).

A oggi, nell'era COVID, la necessità del distanziamento sociale ha evidenziato al massimo il contributo della tecnologia digitale in sanità in termini di accesso alle cure e di spesa sanitaria. In questa epoca, la telemedicina ha supportato alcune categorie di pazienti, in particolare le donne in gravidanza, con problematiche di carattere ginecologico o con malattie infiammatorie croniche intestinali, come riportato da diversi autori (6-8). Nell'ambito della malattia renale cronica, nel cui contesto l'informatizzazione e la tecnologia digitale sono diventate imprescindibili (p. es., monitor di dialisi, dialisi peritoneale, ecc.), la telemedicina rappresenta da sempre uno strumento necessario per migliorare la qualità della vita dei pazienti attraverso soluzioni di autogestione e monitoraggio anche da remoto. L'esempio più calzante è quello relativo alla dialisi peritoneale. La gestione e il monitoraggio a distanza del paziente in dialisi peritoneale è, ormai, un'esperienza acquisita che ha radicalmente cambiato e migliorato non solo la qualità della vita dei pazienti in terapia sostitutiva, ma anche l'aspetto economico (9-11). Tuttavia, esistono altre realtà nel contesto della malattia renale cronica, in cui l'attività di telemonitoraggio può garantire il mantenimento di un'adeguata qualità di vita nei differenti stadi della malattia. Infatti, facendo riferimento al periodo emergenziale, si è resa obbligatoria l'attività di telemedicina dell'ambulatorio nefrologico in virtù del distanziamento sociale, che ha permesso al personale sanitario e ai pazienti di sperimentarne l'efficacia pur in assenza di percorsi procedurali predefiniti. Già diverso tempo prima, grazie all'impegno costante di alcuni gruppi di professionisti dove la "quota rosa" rappresenta la parte più numerosa, è stato possibile, attraverso un progetto ad hoc, verificare la validità della gestione nutrizionale da remoto per pazienti nefropatici in fase conservativa, di cui riportiamo i dati in questo lavoro.

\section{Gestione da remoto del paziente in dialisi peritoneale}

Come è noto, nel 2016, in Italia, il Ministero della Salute ha pubblicato il Piano nazionale delle Cronicità, nel quale si introduceva il concetto di monitoraggio da remoto per determinate patologie croniche, inclusa l'insufficienza renale cronica terminale in trattamento dialitico domiciliare inteso come dialisi peritoneale o emodialisi. Si cominciava a parlare di telemedicina e di tecnologie applicate alla medicina convenzionale allo scopo di implementare, e non sostituire, la visita medica. Gli obiettivi erano quelli di promuovere l'impiego di modelli, tecniche e strumenti della sanità digitale nella gestione della cronicità, al fine di garantire continuità e qualità assistenziale, migliore efficacia, efficienza ed appropriatezza delle cure. Durante la pandemia da COVID-19, I'utilizzo della telemedicina applicata alla dialisi domiciliare, in particolare quella peritoneale, si è rivelata la scelta più adeguata. In effetti, in un recente articolo comparso su PDI (12), tra le raccomandazioni per la gestione del paziente in dialisi peritoneale durante l'emergenza sanitaria a scopo precauzionale, vi è l'incentivo all'utilizzo di dispositivi elettronici che consentano la registrazione e l'invio di immagini e dati biometrici (peso, pressione, immagini dell'exit-site cutaneo del catetere peritoneale) al centro medico di riferimento, per permettere la valutazione a distanza e la risoluzione precoce di problematiche, limitando, così, gli accessi ospedalieri. Inoltre, l'utilizzo della metodologia di controllo in remoto ha permesso di migliorare notevolmente gli aspetti organizzativi e gestionali di questi pazienti, implementando l'attività clinica di tipo pro attivo. Come riportato recentemente da Amici et al. (13) nella gestione del paziente in dialisi peritoneale automatizzata (APD), l'introduzione delle nuove tecnologie di monitoraggio in remoto ha prodotto una significativa riduzione del numero di visite non programmate e dei giorni di ospedalizzazione.

L'esperienza del nostro centro ha confermato quanto detto. Già nel periodo tra marzo e maggio 2020, la tendenza a mantenere il paziente a domicilio e l'utilizzo di metodiche digitali, come videochiamate o invio di materiale elettronico e di parametri biometrici, ci ha consentito una gestione a distanza, grazie anche alla possibilità di utilizzare il controllo in remoto dei trattamenti dialitici in più del $50 \%$ dei casi. Tale controllo aveva le caratteristiche della bidirezionalità cioè la possibilità, da parte dei professionisti sanitari, di verificare le prescrizioni terapeutiche e, se necessario, di modificarle e inviarle al cycler in remoto, ottenendo, al tempo stesso, un feedback da parte del paziente.

Pertanto, l'implementazione della metodologia di teledialisi, in particolare l'utilizzo del monitoraggio in remoto, rappresenta una strategia irrinunciabile, specialmente nell'attuale contesto socio-sanitario, perché permette, di fatto, di annullare le distanze, anche geografiche, tra il paziente e il centro medico, senza necessità di ospedalizzazione o di accessi ripetuti in ospedale. II telemonitoraggio, infine, 
permette al clinico e al paziente di rimanere in contatto diretto, senza trascurare gli aspetti correlati alla relazione terapeutica medico-paziente che, in questo momento storico, assume connotazioni particolarmente rilevanti.

\section{Consulenza dietetica online}

II "Documento di indirizzo per la Malattia Renale Cronica" evidenzia come gli obiettivi di cura nei pazienti con cronicità, non potendo essere rivolti alla guarigione, sono finalizzati al miglioramento del quadro clinico e dello stato funzionale, alla minimizzazione della sintomatologia, alla prevenzione della disabilità e al miglioramento della qualità di vita delle persone con malattia renale cronica (MRC), individuando le azioni più efficaci a partire dagli stadi più precoci della malattia, al fine di ritardare il ricorso alla terapia sostitutiva (14). Per realizzarli e prendere in carico il paziente a lungo termine, è necessario individuare soluzioni innovative che tengano conto di una serie di fattori, tra cui fondamentali sono le risorse umane, la competenza e l'attività correlate alla risposta da fornire alle persone con una malattia cronica. L'attuale situazione sanitaria impone a tutti i professionisti il massimo sforzo per rispondere in modo efficace alle necessità assistenziali, attraverso gli strumenti disponibili, in ottemperanza alle richieste di un'utenza sempre più numerosa e pluripatologica. L'impegno dei dietisti di area nefrologica in tal senso inizia nell'anno 2010, con un progetto il cui obiettivo era rispondere all'esigenza di fornire un Servizio di Consulenza Dietetica online "Renal-diet" (Online). Questo progetto può considerarsi il progetto pilota nell'ambito della consulenza dietetica a distanza per pazienti con malattia renale cronica in Italia, realtà possibile grazie alla collaborazione fra diversi professionisti di area nefrologica attraverso l'istituzione di un board scientifico, composto da rappresentanti della Società Italiana di Nefrologia e dell'Associazione Nazionale Dietisti, che ha effettuato un monitoraggio costante dell'attività svolta da dietiste adeguatamente formate, per garantire ai pazienti, fruitori del servizio, prestazioni che rispondessero a criteri di efficienza ed efficacia (15). La visita in remoto si basava su dati antropometrici di primo livello (peso e statura), necessari per la valutazione nutrizionale, e dati clinici, nonché sugli esami bioumorali che venivano registrati dal nefrologo referente del paziente utilizzando una specifica Web-App, nel rispetto della legge sulla privacy. La valutazione delle abitudini alimentari veniva effettuata dal dietista telefonicamente attraverso la storia dietetica (DH) con il supporto dell'atlante fotografico delle porzioni degli alimenti fornito al paziente durante la visita nefrologica (16-20). Ogni paziente che accedeva al servizio di consulenza dietetica online, dopo la valutazione nutrizionale quali-quantitativa, riceveva un piano dietetico personalizzato. La capacità di coniugare nella giusta misura gli aspetti biologici e psico-sociali della storia dietetica costituisce l'abilità centrale del dietista esperto nel trattamento nutrizionale della MRC. Tale abilità consente l'elaborazione di piani dietetici personalizzati che incontrino sia i gusti sia le necessità del paziente (21). I dati relativi all'attività svolta nel biennio 2011-2013 sono stati oggetto di una tesi del Master in Dietistica Renale svoltosi presso l'Università di Modena e Reggio Emilia, con l'obiettivo di descrivere le caratteristiche cliniche e nutrizionali di un campione di pazienti con IRC afferenti al servizio in remoto. Gli obiettivi dello studio erano i seguenti: 1) descrivere lo stato nutrizionale dei pazienti inviati per la prima volta a un servizio di consulenza dietetica; 2) descrivere il grado di malattia al quale viene consigliata dal nefrologo la terapia nutrizionale dell'IRC; 3) valutare le differenze tra l'alimentazione "normale" e quella "ottimale", anche in relazione allo stadio della patologia. L'analisi di seguito riportata riguarda 285 pazienti con IRC in trattamento conservativo (169 maschi e 116 femmine), distribuiti per regione (Tab. I), con le caratteristiche antropometriche e cliniche riportate nella Tabella II.

I pazienti hanno un BMI mediano di $26,8 \mathrm{~kg} / \mathrm{m}^{2}$ (sovrappeso), mentre il valore mediano di eGFR è di $24,4 \mathrm{ml} / \mathrm{min}$ (stadio $4 \mathrm{NKF}$ ). II range interquartile dimostra, peraltro, una grande variabilità sia del BMI (da 24,2 a $32,0 \mathrm{~kg} / \mathrm{m}^{2}$ ) sia dell'eGFR (da 16,9 a 31,7 mL/min). La Tabella III definisce in maggior dettaglio il quadro clinico dei pazienti.

I pazienti con Insufficienza Renale Cronica (IRC) che accedono al servizio di consulenza dietetica online hanno, nella maggioranza dei casi, numerose comorbidità, quali diabete, obesità e ipertensione (Fig. 1). L'età mediana (71 anni) è compatibile con le poche casistiche disponibili nella popolazione generale. Vi è una prevalenza del sesso maschile in tutte le regioni considerate, forse in relazione con la maggiore incidenza di ipertensione e diabete nei maschi rispetto alle

Tabella I - Provenienza e sesso dei pazienti

\begin{tabular}{lccc}
\hline Provenienza & Maschi & Femmine & Totale \\
\hline Emilia Romagna & 18 & 15 & 33 \\
Sardegna & 48 & 35 & 83 \\
Toscana & 33 & 25 & 58 \\
Veneto & 70 & 41 & 111 \\
\hline Totale & 169 & 116 & 285 \\
\hline
\end{tabular}

Tabella II - Caratteristiche del campione

\begin{tabular}{lccc}
\hline & $\mathbf{P}_{50}$ & $\mathbf{P}_{\mathbf{2 5}}$ & $\mathbf{P}_{\mathbf{7 5}}$ \\
\hline Età (anni) & 71 & 59 & 79 \\
Peso $(\mathrm{kg})$ & 74,0 & 64,5 & 89,0 \\
Statura $(\mathrm{m})$ & 1,7 & 1,6 & 1,7 \\
BMI $\left(\mathrm{kg} / \mathrm{m}^{2}\right)$ & 26,8 & 24,2 & 32,0 \\
Creatinina $(\mathrm{mg} /)$ & 2,5 & 2,0 & 3,5 \\
eGFR $\left(\mathrm{mL} / \mathrm{min} / \mathrm{m}^{2}\right)$ & 24,4 & 16,9 & 31,7 \\
\hline
\end{tabular}

Abbreviazioni: $P=x$ percentile $B M I=$ body mass index e $G F R=$ velocità $d i$ filtrazione glomerulare stimata con la formula MDRD. 
Tabella III - Stato ponderale, funzionalità renale e comorbidità dei pazienti

\begin{tabular}{|c|c|c|}
\hline & $\mathbf{N}$ & $\%$ \\
\hline \multicolumn{3}{|l|}{ Sesso } \\
\hline $\mathrm{F}$ & 116 & 40,7 \\
\hline M & 169 & 59,3 \\
\hline \multicolumn{3}{|l|}{ Età (anni) } \\
\hline$\leq 20$ & 4 & 1,4 \\
\hline $30-39$ & 11 & 3,9 \\
\hline $40-49$ & 16 & 5,6 \\
\hline $50-59$ & 42 & 14,7 \\
\hline $60-69$ & 64 & 22,5 \\
\hline $70-79$ & 78 & 27,4 \\
\hline $80-89$ & 66 & 23,2 \\
\hline$\geq 90$ & 4 & 1,4 \\
\hline \multicolumn{3}{|l|}{ BMI $\left(\mathrm{kg} / \mathrm{m}^{2}\right)$} \\
\hline Sottopeso & 1 & 0,4 \\
\hline Normopeso & 102 & 35,8 \\
\hline Sovrappeso & 83 & 29,1 \\
\hline Obesità classe 1 & 54 & 18,9 \\
\hline Obesità classe 2 & 33 & 11,6 \\
\hline Obesità classe 3 & 12 & 4,2 \\
\hline \multicolumn{3}{|l|}{ GFR (mL/min) } \\
\hline $59-30$ & 80 & 28,1 \\
\hline $29-15$ & 149 & 52,3 \\
\hline$\leq 15$ & 56 & 19,6 \\
\hline Anemia & 174 & 61,1 \\
\hline Iperkaliemia & 62 & 21,8 \\
\hline Diabete mellito tipo 2 & 98 & 34,4 \\
\hline Ipertensione arteriosa & 243 & 85,3 \\
\hline Malattie cardiovascolari & 89 & 31,2 \\
\hline Dislipidemia & 119 & 41,8 \\
\hline Iperuricemia & 109 & 38,2 \\
\hline
\end{tabular}

Abbreviazioni: $P_{x}=B M I=$ body mass index; eGFR = velocità di filtrazione glomerulare stimata con la formula MDRD.

femmine nella popolazione generale. Un'alternativa è che ciò rifletta un bias di selezione da parte dei nefrologi o di disponibilità dei pazienti ad accedere al servizio online. Il dato relativo alle comorbidità è particolarmente rilevante. Nell' $85 \%$ dei pazienti si riscontra ipertensione arteriosa e il 31,2\% di essi presenta anche una cardiopatia. L'anemia si riscontra nel $61,1 \%$ del campione, il diabete nel $34,4 \%$ e la dislipidemia nel $41,8 \%$ dei pazienti. Altre alterazioni frequenti sono l'iperpotassiemia e l'iperuricemia, che contribuiscono a complicare la gestione terapeutica e nutrizionale dei soggetti, come riportato nella Figura 1.

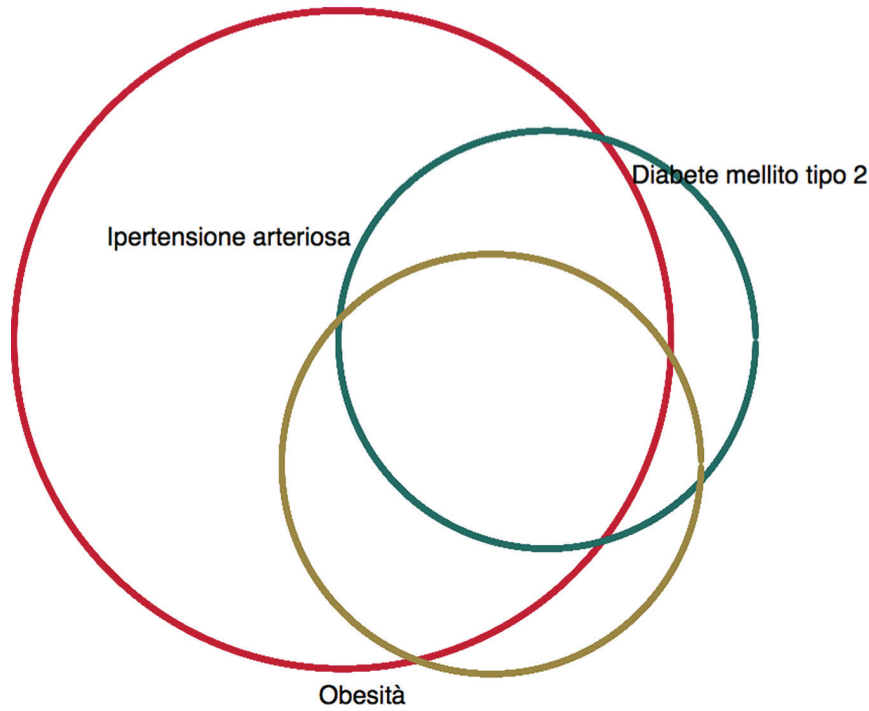

Fig. 1 - Diagramma di Venn della clusterizzazione di ipertensione arteriosa (in rosso), diabete mellito di tipo 2 (in verde) e obesità (in giallo).

Questa Figura dimostra non solo la prevalenza dell'ipertensione arteriosa sul diabete e sull'obesità, ma, soprattutto, come queste condizioni si intersechino tra loro definendo quadri clinici molto variabili di pluripatologia ed evidenziando il fatto che la quasi totalità dei pazienti obesi e diabetici è ipertesa. Un dato molto interessante riguarda il BMI, che è un indicatore attendibile di malnutrizione, sia per difetto che per eccesso. Nonostante il grado mediano di patologia renale sia elevato (eGFR $=24,4 \mathrm{~mL} / \mathrm{min}$ ), la maggioranza dei pazienti è malnutrita per eccesso. Infatti, non solo la mediana del BMI è pari a $26,8 \mathrm{~kg} / \mathrm{m}^{2}$, ma, inoltre, il $29,1 \%$ dei pazienti è in sovrappeso, il $18,9 \%$ ha un'obesità di grado I, I' $11,6 \%$ un'obesità di grado II e il 4,2\% un'obesità di grado III. II sottopeso è praticamente inesistente, essendo pari allo $0,4 \%$. Analizzando in dettaglio il grado di IRC dei pazienti, si rileva che il $28,1 \%$ di essi è allo stadio 3 NKF, il 52,3\% allo stadio 4 e il 19,6\% allo stadio 5. Le abitudini alimentari dei pazienti all'ingresso nel programma sono evidenziate nella Tabella IV.

L'anamnesi alimentare evidenzia che la quota calorica mediana prima del trattamento dietetico è pari a $2.115 \mathrm{kcal} /$ die, mentre la mediana dell'introito proteico corrisponde a $70 \mathrm{~g} /$ die e a $0,9 \mathrm{~g} / \mathrm{kg} /$ peso corporeo, con una prevalenza di proteine animali (60\%). Per quanto riguarda i lipidi, si riscontra una mediana di $85 \mathrm{~g}$, di cui i saturi rappresentano il $29 \%$. La quota mediana di carboidrati è pari a $265 \mathrm{~g} /$ die e gli zuccheri semplici rappresentano il 31\% dei carboidrati totali. L'introito mediano di fibra è pari a $22 \mathrm{~g} /$ die e quello di colesterolo equivale a $215 \mathrm{mg} / \mathrm{die}$. Il sodio introdotto con gli alimenti è pari a $1.767 \mathrm{mg} / \mathrm{die}$ al $50^{\circ}$ percentile. Non è stato possibile stimare il sodio totale a causa dell'impossibilità di valutare la quantità di cloruro di sodio aggiunto dai pazienti. La mediana dell'introito di potassio, stimato considerando i soli alimenti, è pari a $2.910 \mathrm{mg} / \mathrm{die}$. La quantità mediana di fosforo introdotta 
Tabella IV - Assunzione di nutrienti all'ingresso nel programma

\begin{tabular}{lccc}
\hline & $\mathbf{P}_{\mathbf{5 0}}$ & $\mathbf{P}_{\mathbf{2 5}}$ & $\mathbf{P}_{\mathbf{7 5}}$ \\
\hline E (Kcal/die) & 2.115 & 1.812 & 2.465 \\
PRO (g/die) & 70 & 56 & 85 \\
PROA (\% PRO) & 60 & 53 & 72 \\
PRO (g/kg peso) & 0,9 & 0,8 & 1,1 \\
FAT (g) & 85 & 70 & 101 \\
SFA (\% FAT) & 29 & 26 & 34 \\
CHO (g) & 265 & 214 & 320 \\
CHOS (\% CHO) & 31 & 26 & 38 \\
FIB (g/die) & 22 & 18 & 27 \\
CH (mg/die) & 215 & 163 & 267 \\
Na (mg/die) & 1.767 & 1.288 & 2.342 \\
K (mg/die) & 2.910 & 2.321 & 3.317 \\
P (mg/die) & 1.115 & 913 & 1.377 \\
Etanolo (g/die) & 3 & 0 & 16 \\
\hline
\end{tabular}

Abbreviazioni: $P_{x}=x$ percentile; $E=$ Energia; $P R O=$ proteine; $P R O A=$ proteine animali; $F A T=$ grassi; $S F A=$ grassi saturi; $\mathrm{CHO}=$ carboidrati; $\mathrm{CHOS}=$ carboidrat complessi; $F I B=$ fibra; $\mathrm{CH}=$ colesterolo; $\mathrm{Na}=$ sodio; $\mathrm{K}=$ potassio; $P=$ fosforo.

giornalmente è pari a $1.115 \mathrm{mg} / \mathrm{die}$. II consumo di etanolo è decisamente basso, con una mediana di $3 \mathrm{~g} / \mathrm{die}$, variando da un consumo nullo al $25^{\circ}$ percentile e pari a $16 \mathrm{~g} / \mathrm{die}$ al $75^{\circ}$ percentile. Durante I'anamnesi alimentare, condotta con un intento quali-quantitativo, i pazienti riferiscono di aver ridotto i nutrienti critici, e questo può essere vero, se si prende in considerazione il livello medio di assunzione delle proteine nella popolazione occidentale. Tuttavia, hanno ricevuto indicazioni generiche e non motivate per cui hanno allestito una dieta "fai-da-te", dove, per ridurre o eliminare alimenti "proibiti", ne introducono di nuovi altrettanto sconsigliati. Riportiamo nella Tabella $\mathrm{V}$ le più frequenti indicazioni

Tabella V - Indicazioni più frequenti Vs comportamenti dei pazienti

- "Mangiare poca carne", con il risultato che il paziente elimina la carne e aumenta la frequenza di consumo dei formaggi "perché hanno poche proteine"

- "Eliminare completamente i latticini e i formaggi", a eccezione di formaggi come la mozzarella, la ricotta e le sottilette, che spesso dai pazienti non sono considerati tali

- "Eliminare completamente i legumi", perché contengono le proteine e, di conseguenza, chi consumava pasta e fagioli aumenta la frequenza settimanale di condimenti a base di carne

- "Sostituire i prodotti aproteici, se non sono graditi, con il riso", variazione che comporta un aumento dell'assunzione proteica

- "Evitare frutta e verdura" a causa dell'iperkaliemia, senza considerare che l'eliminazione di alimenti determina una riduzione dell'apporto energetico

- "Eliminare il sale dalla dieta", senza considerare che la palatabilità degli alimenti incide in modo rilevante sulla riduzione dell'appetito con conseguente riduzione dell'apporto energetico
Tabella VI - Bromatologia del piano dietetico personalizzato

\begin{tabular}{lccc}
\hline & $\mathbf{P}_{\mathbf{5 0}}$ & $\mathbf{P}_{\mathbf{2 5}}$ & $\mathbf{P}_{\mathbf{7 5}}$ \\
\hline E (Kcal/die) & 1.858 & 1.679 & 2.011 \\
PRO (g/die) & 44 & 40 & 51 \\
PROA (\% PRO) & 54 & 48 & 58 \\
PRO (g/kg peso) & 0,6 & 0,5 & 0,7 \\
FAT (g) & 62 & 56 & 69 \\
SFA (\% FAT) & 29 & 27 & 31 \\
CHO (g) & 277 & 247 & 308 \\
CHOS (\% CHO) & 22 & 20 & 25 \\
FIB (g/die) & 26 & 24 & 29 \\
CH (mg/die) & 133 & 120 & 145 \\
Na (mg/die) & 1.300 & 1.150 & 1.500 \\
K (mg/die) & 2.096 & 1.950 & 2.290 \\
P (mg/die) & 715 & 641 & 800 \\
\hline
\end{tabular}

Abbreviazioni: $P_{x}=x_{\text {esimo }}$ percentile; $E=$ Energia; $P R O=$ proteine; $P R O A=$ proteine animali; $F A T=$ grassi; $S F A=$ grassi saturi; $C H O=$ carboidrati; $C H O S=$ carboidrati complessi; $F I B=$ fibra; $\mathrm{CH}=$ colesterolo; $\mathrm{Na}=$ sodio; $\mathrm{K}=$ potassio; $P=$ fosforo.

ricevute dai pazienti prima dell'incontro con il dietista e la loro interpretazione/applicazione all'alimentazione usuale.

In base alla storia dietetica e alle Linee Guida di riferimento (22), a seguito della valutazione del quadro clinico riportato dal medico nefrologo, viene formulato un piano dietetico personalizzato, in accordo con il paziente e la famiglia, di cui si riporta nella Tabella VI la bromatologia.

In generale, i piani dietetici elaborati hanno un apporto energetico e proteico inferiore, mantenendo un apporto di proteine animali pari al $50 \%$ della quota proteica totale. Sono più equilibrati fosforo, sodio, potassio, grassi, colesterolo e zuccheri. Qualora sia presente ritenzione idrica con rischio di scompenso cardiaco, vengono fornite le indicazioni per la riduzione del sodio ( $<100 \mathrm{mEq} /$ die). Particolare attenzione viene posta alla valutazione delle abitudini quali-quantitative dell'alimentazione e alle preferenze del paziente, ogni volta che vengono eliminati o inseriti un nuovo alimento o un gruppo di alimenti o un prodotto dietetico, motivando le modifiche dell'alimentazione dettate dalla situazione clinica. La Figura 2 mostra le differenze tra l'alimentazione usuale riportata dal paziente/famiglia e quella consigliata dal dietista.

I valori riportati sono mediane con intervalli di confidenza al $95 \%$. II confronto tra le mediane è stato effettuato utilizzando la regressione quantile. Da questa Figura si rileva come I'introito di energia sia stato ridotto, così come l'introito proteico e di grassi, e come, invece, si siano ridotti i carboidrati semplici e sia stata aumentata la fibra rispetto alla dieta abituale dei pazienti. Più specificatamente, si è passati da una mediana di $2.115 \mathrm{kcal} /$ die a una di $1.859 \mathrm{kcal} / \mathrm{die}$, da $70 \mathrm{~g} / \mathrm{die}$ a $44 \mathrm{~g} /$ die di proteine (proteine animali dal $60 \%$ al 54\%), pari a $0,6 \mathrm{~g} / \mathrm{kg} / \mathrm{die}$ contro gli $0,9 \mathrm{~g} / \mathrm{kg} / \mathrm{die}$ abituali. Analogo 


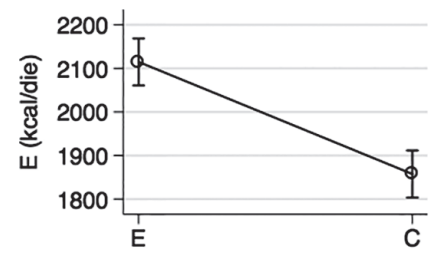

$p<0.001$

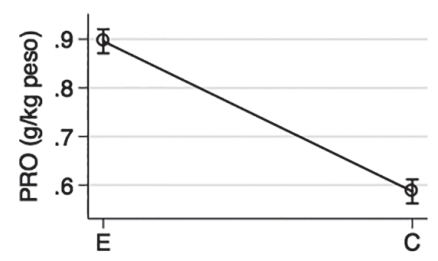

$p<0.001$

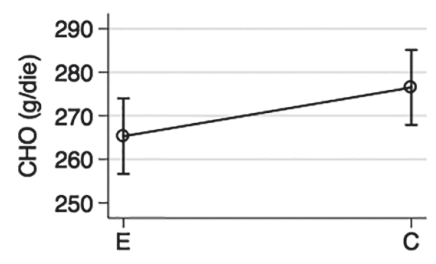

$p=0.073$

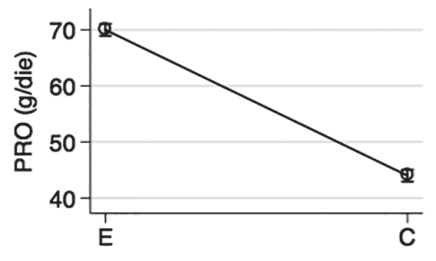

$p<0.001$

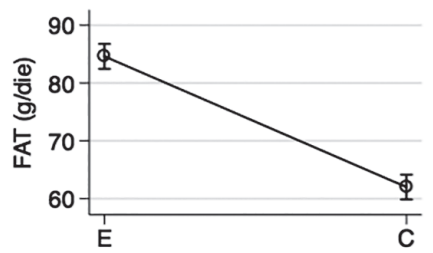

$p<0.001$

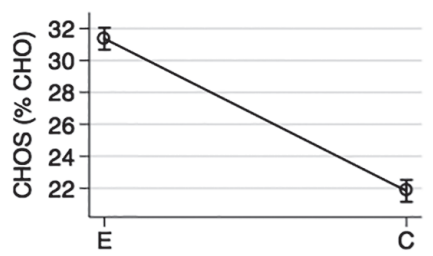

$p<0.001$
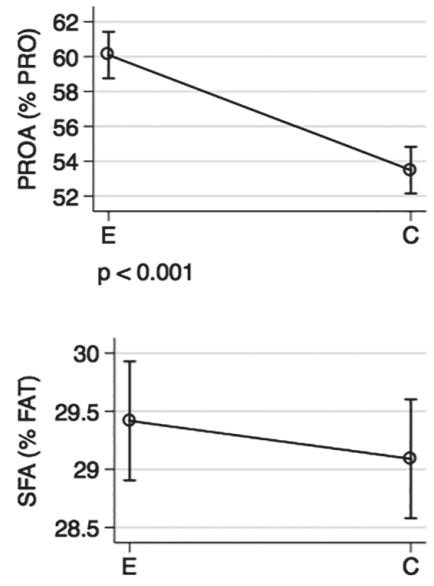

$p=0.376$

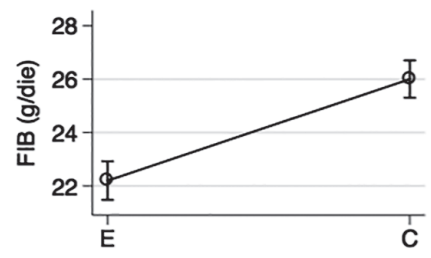

$\mathrm{p}<0.001$
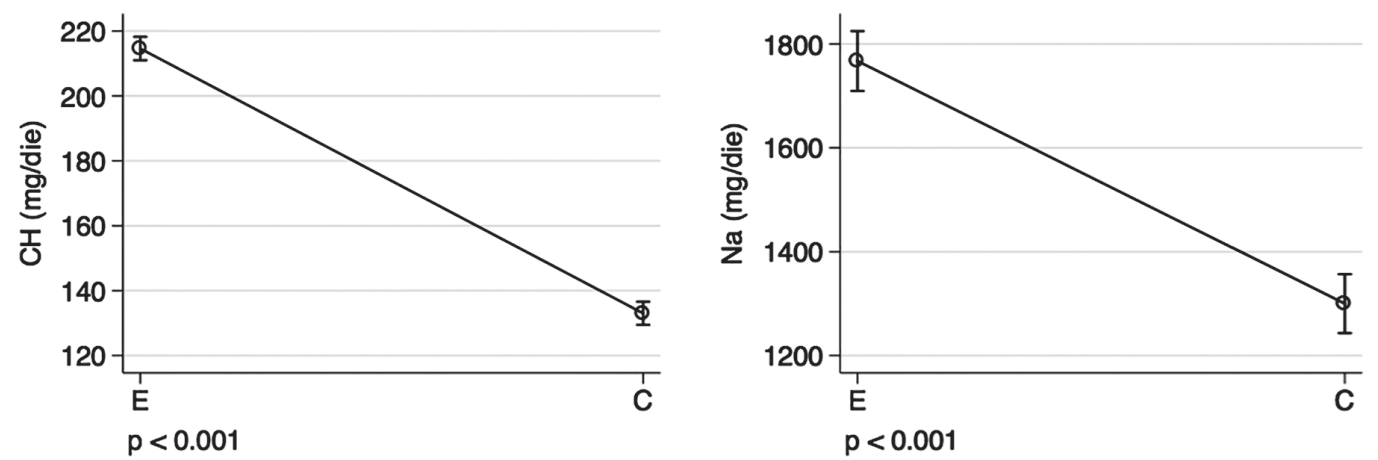

Fig. 3 - Differenza tra assunzione effettiva e consigliata di nutrienti.

Abbreviazioni: $E=$ assunzione effettiva; $\mathrm{C}=$ assunzione consigliata; $\mathrm{CH}=$ colesterolo; $\mathrm{Na}=$ sodio; $K=$ potassio; $P=$ fosforo. $I I$ confronto tra le mediane è stato effettuato utilizzando la regressione quantile.

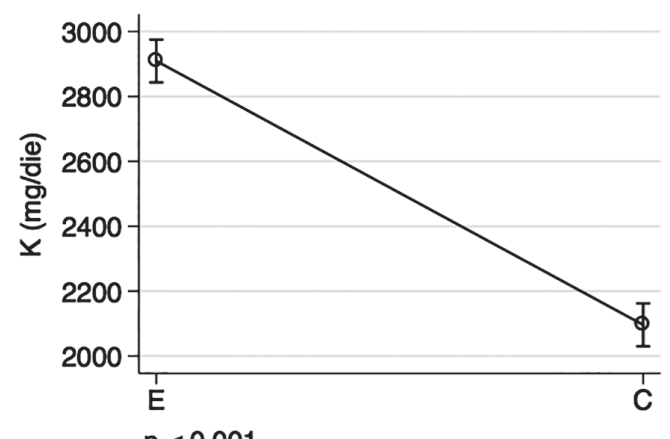

$p<0.001$

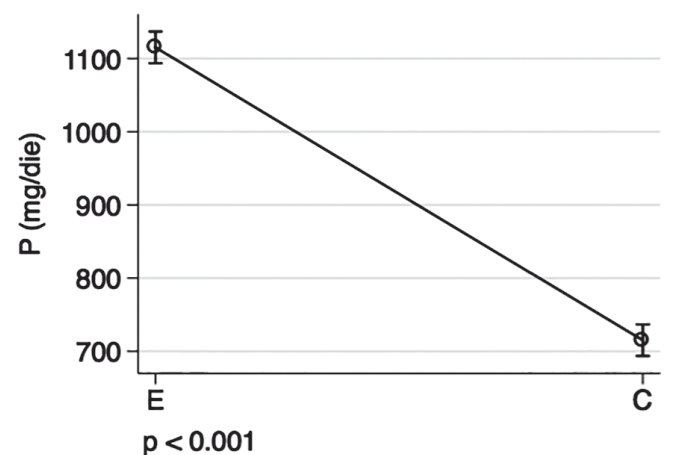

Fig. 2 - Differenza tra assunzione effettiva e consigliata di Abbreviazioni: $E=$ assunzione

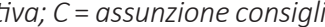
ata; $E=$ Energia; $P R O=$ proteine,
$P R O A=$ proteine animali $;$ FAT grassi; SFA = grassi saturi; $\mathrm{CHO}=$ complessi; FIB = fibra. comportamento per i lipidi, infatti si è passati dagli 85 g/die ai $60 \mathrm{~g} /$ die come media, con stabilità sostanziale della quota dei saturi. La quantità di fibra consigliata registra un aumento, passando da una mediana di $22 \mathrm{~g} /$ die a $26 \mathrm{~g} /$ die, altro dato che attesta come la qualità della dieta sia migliorata.
Modificazioni sostanziali si sono verificate anche per i micronutrienti (Fig. 3).

Anche in questo caso, si rileva come, prima di un percorso educativo che richiede frequenti contatti con il professionista, le assunzioni di nutrienti effettive si discostino da quelle 
consigliate. Si nota, infatti, una diminuzione della mediana del colesterolo da $215 \mathrm{mg} /$ die a $133 \mathrm{mg} /$ die. II sodio da alimenti si riduce, passando da $1.767 \mathrm{mg} /$ die a $1.330 \mathrm{mg} / \mathrm{die}$, e lo stesso vale sia per il potassio, che passa da $2.910 \mathrm{mg} / \mathrm{die}$ a $2.096 \mathrm{mg} /$ die, che per il fosforo, che si riduce da $1.115 \mathrm{mg} /$ die a $715 \mathrm{mg} /$ die, rientrando nell'ambito dei valori raccomandati per i pazienti con IRC in fase conservativa. Le modificazioni di questi micronutrienti sono centrali per la gestione nutrizionale della malattia renale (23-26). Si sottolinea che i pazienti afferenti al servizio di consulenza dietetica online non sono stati arruolati per uno studio dettagliato e volto a rilevare outcome di interesse clinico, quindi le considerazioni effettuate su questo campione di pazienti non sono necessariamente estendibili a popolazioni differenti. L'esperienza descritta, svoltasi in un periodo pre-COVID, ha dimostrato come la consulenza dietetica in remoto possa offrire ai pazienti con malattia renale cronica un'ulteriore e preziosa possibilità di "cura", al fine di raggiungere e/o mantenere un adeguato stato nutrizionale e, inoltre, attraverso un follow-up periodico, garantire un adeguato atteggiamento terapeutico nel tempo a tutela della salute.

\section{Conclusioni}

L'emergenza sanitaria COVID-correlata ha evidenziato I'importanza della telemedicina, che il progresso tecnologico aveva già introdotto attraverso sistemi di monitoraggio dei pazienti a distanza a partire dall'anno 1976. Da allora, vari enti di ricerca, con l'ausilio del Ministero della Salute, hanno messo a punto diversi progetti, che hanno dato origine alla crescita esponenziale dei servizi disponibili.

Anche in ambito nefrologico esistono vari campi di applicazione della telemedicina, che consentono non solo di contenere le visite ambulatoriali in presenza, ma, al tempo stesso, di garantire una buona relazione terapeutica attraverso la promozione dell'autonomia, del grado di consapevolezza e della gestione responsabile del programma da parte del paziente. Per quanto riguarda la dialisi peritoneale, esperienze di diversi centri italiani (13) hanno mostrato come, con il ricorso alla telemedicina, si sono addirittura ridotti non solo il numero delle visite non programmate ma anche i giorni di ospedalizzazione. Per quanto riguarda il trattamento dietetico in remoto, grazie al contributo di professionisti competenti, all'utilizzo di supporti adeguati e alla disponibilità di pazienti motivati, è stato possibile proporre un percorso nutrizionale personalizzato che necessita nel tempo di un monitoraggio adeguato. In conclusione, l'emergenza sanitaria ha necessariamente implementato la telemedicina anche per i pazienti nefropatici, rinforzando l'utilizzo di metodiche già in uso in alcuni campi specifici del settore. La vera sfida della telemedicina sarà valutarne l'efficienza e l'efficacia a lungo termine e comprendere se tale tecnica può assumere lo "status" di attività routinaria a integrazione di quella tradizionale.

\section{Disclosures}

Authorship: All authors contributed equally.

Conflict of interest: The authors declare no conflict of interest.

Financial support: This research received no specific grant from any funding agency in the public, commercial, or not-for-profit sectors.

\section{Bibliografia}

1. Strategia per la crescita digitale 2014-2020. Presidenza del Consiglio dei Ministri. Versione pubblicata il 21 giugno 2016. Online (Accessed December 15, 2020)

2. Telemedicina. Enciclopedia Treccani. Disponibile online in Online (Accessed December 15, 2020)

3. Maceratini R, Ricci F. Il medico online. Manuale di informatica medica. Verduci Editore. 2001 (Accessed December 15, 2020)

4. National Strategy for e-health - Sweden. Disponibile online in Online (Accessed December 15, 2020)

5. Ministero della Salute. Telemedicina Linee di Indirizzo Nazionali. Data di pubblicazione 17/03/2014. Online (Accessed December 15, 2020)

6. Cohen MA, Powell AM, Coleman JS, Kelle JM, Livingston A, Anderson JR. Special Ambulatory Gynecologic Considerations in the Era of COVID-19 and Implications for Future Practice. Am J Obstet Gynecol. 2020 Jun 6;S0002-9378(20):30621-30629. CrossRef PubMed

7. Murphy HR. Managing Diabetes in Pregnancy Before, During, and After COVID-19. Diabetes Technol Ther. 2020;22(6): 454-461. CrossRef PubMed

8. Siniscalchi $M$, Zingone $F$, Savarino EV, D'Odorico A, Ciacci C. COVID-19 Pandemic Perception in Adults With Celiac Disease: An Impulse to Implement the Use of Telemedicine: COVID-19 and CeD. Dig Liver Dis. 2020;52(10):1071-1075. CrossRef

9. Makhija D, Alscher MD, Becker S, et al. Remote Monitoring of Automated Peritoneal Dialysis Patients: Assessing Clinical and Economic Value. Telemed J E Health. 2018 Apr;24(4):315-323. CrossRef Epub 2017 Oct 12

10. Nayak KS, Ronco C, Karopadi AN, Rosner MH. Telemedicine and Remote Monitoring: Supporting the Patient on Peritoneal Dialysis. Perit Dial Int. 2016;36(4):362-366. CrossRef PubMed

11. Magnus M, Sikka N, Cherian T, Lew SQ. Satisfaction and Improvements in Peritoneal Dialysis Outcomes Associated with Telehealth. Appl Clin Inform. 2017;8(1):214-225. CrossRef PubMed

12. Ronco C, Manani SM, Giuliani A, Tantillo I, Reis T, Brown EA. Remote patient management of peritoneal dialysis during COVID-19 pandemic. Perit Dial Int. 2020;40(4):363-367. CrossRef PubMed

13. Amici $G$. The advantages of remote patient monitoring in automated peritoneal dialysis. G Ital Nefrol. 2020;37(3):2020vol3. PubMed

14. Ministero della Salute. Documento di indirizzo per la Malattia Renale Cronica, 05/08/2014. Online (Accessed December 15, 2020)

15. Wiggins KL, Harvey KS. A review of guidelines for nutrition care of renal patients. J Ren Nutr 2002; 12:190-6 CrossRef PubMed

16. Fantuzzi AL, Chiuchiù MP, Bedogni G. Atlante fotografico delle porzioni degli alimenti. Milano: Istituto Scotti Bassani; 2005. (Accessed December 15, 2020)

17. Posizione ANDID. La gestione nutrizionale del paziente con Malattia Renale Cronica. ANDID; 2004. (Accessed December 15, 2020)

18. Fantuzzi AL, Bedogni G. Dieta ipoproteica e insufficienza renale cronica: manuale pratico per il dietista. Milano UTET; 2003. (Accessed December 15, 2020) 
19. Dwyer JT. Dietary assessment. In: Shils ME, Olson JA, Shike M, eds. Modern Nutrition in health and disease. Philadelphia: Lea \& Fabriger; 1994:842-860.

20. Livingstone MB, Robson PJ. Measurement of dietary intake in children. Proc Nutr Soc. 2000;59(2):279-293. CrossRef PubMed

21. Kopple JD. Nutrition, diet and the kidney. In "Modern nutrition in health and disease", Shils ME, Olson JA, Shike M. Lea and Febiger, Philadelphia. 1994:1102-1134.

22. Fouque D. Vennegor M, Wee $P$ et al. European Best Practice Guidelines (EBPG). Nephrol Dial Transplant. 2007;22(suppl 2): ii45-ii87. CrossRef PubMed

23. Barsotti G, Cupisti A. The role of dietary phosphorus restriction in the conservative management of chronic renal disease. J Ren Nutr. 2005;15(1):189-192. CrossRef PubMed
24. Gross JL, de Azevedo MJ, Silveiro SP, Canani LH, Caramori ML, Zelmanovitz T. Diabetic nephropathy: diagnosis, prevention, and treatment. Diabetes Care. 2005;28(1):164-176. CrossRef PubMed

25. Gin H, Rigalleau V, Aparicio M. Lipids, protein intake, and diabetic nephropathy. Diabetes Metab. 2000;26(suppl 4): 45-53. PubMed

26. Cianciaruso B, Bellizzi V, Brunori G, et al. La dieta ipoproteica oggi in italia: le conclusioni del gruppo di lavoro. G Ital Nefrol. 2008;25(S-42):S54-S57. 